\title{
LncRNA and transcriptomic analysis of fetal membrane reveal potential targets involved in oligohydramnios
}

Yu-hua Ou ${ }^{1,2 \dagger}$, Yu-kun Liü ${ }^{2 \dagger}$, Li-qiong Zhu ${ }^{2}$, Man-qi Chen ${ }^{2}$, Xiao-chun Yi $^{2}$, Hui Chen ${ }^{2^{*}}$ and Jian-ping Zhang ${ }^{2^{*}}$

\begin{abstract}
Background: The multiple causes of oligohydramnios make it challenging to study. Long noncoding RNAs (IncRNAs) are sets of RNAs that have been proven to function in multiple biological processes. The purpose of this study is to study expression level and possible role of IncRNAs in oligohydramnios.

Methods: In this study, total RNA was isolated from fetal membranes resected from oligohydramnios pregnant women (OP) and normal amount of amniotic fluid pregnant women (Normal). LncRNA microarray was used to analyze the differentially expressed IncRNAs and mRNAs. Kyoto Encyclopedia of Genes and Genomes (KEGG) was used to analyze the main enrichment pathways of differentially expressed mRNAs. Real-time quantitative PCR (qPCR) was used to validate the IncRNA expression level.

Results: LncRNA microarray analysis revealed that a total of 801 IncRNAs and 367 mRNAs were differentially expressed in OP; in these results, 638 IncRNAs and 189 mRNAs were upregulated, and 163 IncRNAs and 178 mRNAs were downregulated. Of the IncRNAs, 566 were intergenic IncRNAs, 351 were intronic antisense IncRNAs, and 300 were natural antisense IncRNAs. The differentially expressed IncRNAs were primarily located in chromosomes 2, 1, and 11. KEGG enrichment pathways revealed that the differentially expressed mRNAs were enriched in focal adhesion as well as in the signaling pathways of Ras, tumor necrosis factor (TNF), estrogen, and chemokine. The qPCR results confirmed that LINC00515 and RP11-388P9.2 were upregulated in OP. Furthermore, the constructed IncRNA-miRNA-mRNA regulatory network revealed tenascin R (TNR), cystic fibrosis transmembrane conductance regulator (CFTR), ATP-binding cassette sub-family A member 12 (ABCA12), and collagen 9A2 (COL9A2) as the candidate targets of LINC00515 and RP11-388P9.2.
\end{abstract}

Conclusions: In summary, we revealed the profiles of IncRNA and mRNA in OP. These results might offer potential targets for biological prevention for pregnant women with oligohydramnios detected before delivery and provided a reliable basis for clinical biological treatment in OP.

Keywords: Biological process, Regulatory network, Fetal membrane, Oligohydramnios

\footnotetext{
*Correspondence: chenhui9@mail.sysu.edu.cn; zhjianp@mail.sysu.edu.cn

†Yu-hua Ou and Yu-kun Liu contributed equally to this work.

2 Department of Obstetrics and Gynecology, Sun Yat-sen Memorial Hospital,

Sun Yat-sen University, No.107, Yanjiangxi Road, Guangzhou 510120,

Guangdong, China

Full list of author information is available at the end of the article
}

(c) The Author(s). 2020 Open Access This article is licensed under a Creative Commons Attribution 4.0 International License, which permits use, sharing, adaptation, distribution and reproduction in any medium or format, as long as you give appropriate credit to the original author(s) and the source, provide a link to the Creative Commons licence, and indicate if changes were made. The images or other third party material in this article are included in the article's Creative Commons licence, unless indicated otherwise in a credit line to the material. If material is not included in the article's Creative Commons licence and your intended use is not permitted by statutory regulation or exceeds the permitted use, you will need to obtain permission directly from the copyright holder. To view a copy of this licence, visit http://creativecommons.org/licenses/by/4.0/ The Creative Commons Public Domain Dedication waiver (http://creativecommons.org/publicdomain/zero/1.0/) applies to the data made available in this article, unless otherwise stated in a credit line to the data. 


\section{Background}

Amniotic fluid is critical for a healthy pregnancy because it allows for fetal movements and it protects the fetus from trauma by acting as a physical cushion. It also plays an important role in fetal lung and limb development [1]. The volume of amniotic fluid varies at different stages of pregnancy [2,3], and the average amniotic fluid volume is $400 \mathrm{~mL}$ at term [4].

Oligohydramnios is generally defined as a reduced amount of amniotic fluid. Amniotic fluid volume in the third trimester of pregnancy with less than $300 \mathrm{~mL}$, amniotic fluid index $(\mathrm{AFI})<5 \mathrm{~cm}$, and single deepest pocket (SDP) $\leq 2 \mathrm{~cm}$ [5-8] are the commonly used parameters for diagnosis. Oligohydramnios is a common complication during pregnancy; it can increase delivery rates and labor induction rates in pregnant women and can significantly increase the mortality rate of perinatal children $[9,10]$. In addition, oligohydramnios is associated with intrauterine fetal growth restriction and prolonged labor [11].

Currently, the mechanisms underlying oligohydramnios remain unclear. Long noncoding RNA (lncRNA) is a class of transcripts that contains more than 200 nucleotides, but it cannot encode proteins. LncRNAs have been proven to be expressed in a wide range of diseases, and they are involved in regulating cancer development and metastasis [12], heart diseases [13, 14], and autoimmune diseases [15]. There is also evidence suggesting that lncRNA is involved in pregnancy-associated events. For example, placental lncRNA expression is alert in response to phthalate exposure during pregnancy [16]. Furthermore, lncRNA uc003fir suppresses the proliferation and migration of trophoblast cells, which might contribute to preeclampsia development [17]. However, little is known about the association between lncRNAs and oligohydramnios.

Therefore, in the present study, we performed lncRNA and mRNA microarray analyses to explore the lncRNA and mRNA expression profile in response to oligohydramnios in pregnant women. Both lncRNAs and mRNAs were sequenced for IncRNA-miRNA-mRNA integrated analysis. In this study, we provided the first evidence that lncRNAs and mRNAs were differentially expressed in the fetal membrane in oligohydramnios pregnant women (OP); based on the IncRNA-miRNAmRNA network, we predicted the potential role of lncRNAs and mRNAs in OP.

\section{Methods}

\section{Patient recruitment}

We conducted a retrospective cohort study of pregnant women with oligohydramnios in Sun Yat-sen Memorial Hospital. In 2017, the number of deliveries in the Obstetric Department of Sun Yat-sen Memorial Hospital was 2667. Among them, 45 cases were pregnant women with oligohydramnios. Due to emergency cesarean section of pregnant women, part of fetal membrane tissues was lost due to failure to freeze in liquid nitrogen within 15 min. Finally, the fetal membrane tissues of 20 pregnant women with oligohydramnios and 19 normal amount of amniotic fluid pregnant women (Normal) were collected and used in this study. The age of all pregnant women was between 21 and 37 years. Both groups of pregnant women had no secondary diseases, intrauterine infection, smoking, alcohol, fetal developmental abnormalities, acute chorioamnionitis, premature rupture of membranes, and drugs used, including angiotensin converting enzyme inhibitor, angiotensin II receptor blockers, non-steroidal anti-inflammatory drugs during pregnancy. The blood pressure in the two groups of pregnant women was within the normal range.

\section{Diagnostic criteria for oligohydramnios}

Pregnant women who meet the following standard criteria are diagnosed with oligohydramnios: amniotic fluid volume in the third trimester of pregnancy with less than $300 \mathrm{~mL}$, an SDP of $\leq 2 \mathrm{~cm}$ or an AFI of $\leq 5 \mathrm{~cm}$ [58]. Simultaneously, when the AFI is less than $8 \mathrm{~cm}$, it is considered to be less amniotic fluid volume [18]. The mean values of the AFI and SDP for pregnant women with oligohydramnios, which were detected and diagnosed by the same sonographer through ultrasound and the pregnant women with oligohydramnios were reexamined by ultrasound again at the internal of 2-4 days, were $53.81 \pm 13.82$ and $26.95 \pm 7.51 \mathrm{~mm}$, respectively. And the content of amniotic fluid estimated by the obstetrician in pregnant women with oligohydramnios during delivery was about $194.29 \pm 50.06 \mathrm{~mL}$, which was less than $300 \mathrm{~mL}$. The fetal membrane tissues of pregnant women with AFI $\leq 5 \mathrm{~cm}$ were used for microarray analysis. The fetal membrane tissues of pregnant women with AFI of $53.81 \pm 13.82 \mathrm{~mm}$ and SDP of $26.95 \pm 7.51$ $\mathrm{mm}$ were used for real-time quantitative PCR (qPCR) verification. Simultaneously, the obstetric outcomes (Table 1) and pregnancy complications (Table 2) of pregnant women were also analyzed.

\section{Tissue collection}

When giving birth in an operating room or a maternity room, after the placentas were delivered, the fetal membrane tissues $2 \mathrm{~cm}$ from the periphery of the umbilical cord were cut. The cut fetal membrane tissues, which were washed with sterile phosphate buffered saline (PBS) and then dried with sterile gauze, were about 4 $\mathrm{cm}^{*} 4 \mathrm{~cm}$ in size. All samples were immediately frozen in liquid nitrogen within $15 \mathrm{~min}$ and then transferred to $80{ }^{\circ} \mathrm{C}$ refrigerator for storage for later use. 
Table 1 Demographic information

\begin{tabular}{|c|c|c|c|}
\hline Characteristics & Oligohydramnios $(n=20)$ & $\begin{array}{l}\text { Normal control } \\
(n=19)\end{array}$ & P-value \\
\hline Age (years) & $32.63 \pm 5.23$ & $30.8 \pm 4.17$ & 0.233 \\
\hline Weeks of delivery & $37.34 \pm 2.01$ & $39.84 \pm 2.88$ & $0.003^{*}$ \\
\hline $\mathrm{BMl}$ & $26.21 \pm 2.48$ & $26.23 \pm 2.81$ & 0.977 \\
\hline Number of pregnancy & $2.48 \pm 1.50$ & $2.68 \pm 1.62$ & 0.68 \\
\hline Number of productions & $1.38 \pm 0.50$ & $1.47 \pm 0.88$ & 0.695 \\
\hline Total number of abortions & $1.09 \pm 1.34$ & $1.21 \pm 1.15$ & 0.775 \\
\hline Spontaneous abortion & $0.16 \pm 0.37$ & $0.7 \pm 0.92$ & $0.023^{*}$ \\
\hline IVF (\%) & $2(9.5)$ & $1(5.2)$ & 0.538 \\
\hline Apgar 1 min & $9.65 \pm 0.74$ & $9.89 \pm 0.31$ & 0.131 \\
\hline \multicolumn{4}{|l|}{ Baby gender } \\
\hline Male & 8 & 8 & - \\
\hline Female & 12 & 11 & - \\
\hline Delivery method & & & 0.072 \\
\hline Normal delivery (\%) & $8(4)$ & 13(68.4) & \\
\hline Cesarean section (\%) & $12(60)$ & $6(31.6)$ & \\
\hline Birth weight (kg) & $2.67 \pm 0.57$ & $3.31 \pm 0.23$ & $3.89 \mathrm{E}-5^{*}$ \\
\hline Placental weight (kg) & $0.47 \pm 0.04$ & $0.51 \pm 0.02$ & $0.003^{*}$ \\
\hline
\end{tabular}

BMI Body Mass Index, IVF In-vitro fertilization. ${ }^{*} P<0.05$ (Student's t-test). Variable is mean \pm standard deviation

\section{RNA isolation}

The membranes were washed prior to being homogenized. Approximately $1 \mathrm{~cm}^{3}$ of the tissue block was resected for grinding. Samples were ground in a motordriven homogenizer. Trizol (Invitrogen, CA, USA) was used to extract total RNA from the tissues in accordance with the manufacturer's protocol. The concentration and qualification of the isolated total RNA was assessed by a Nanodrop 2001 spectrophotometer (Thermo Fisher Scientific, MA, USA).

\section{LncRNA and mRNA microarray analysis}

Total RNA from the fetal membranes, which were obtained from five $\mathrm{OP}$ and five Normal, were used for microarray analysis. The Human LncRNA Array V4.0 $(8 \times 60 \mathrm{k})$ was performed by KangChen Bio-tech Inc. (Shanghai, China) according to the manufacturer standard protocols. The microarray analyses included 40,173 lncRNAs and 20,730 mRNAs.

\section{Bioinformatics}

Agilent Feature Extraction software (version 11.0.1.1) was used to analyze the acquired array images. Quantile normalization and subsequent data processing were performed with the GeneSpring GX v11.5.1 software package (Agilent Technologies). Differentially expressed lncRNAs and mRNAs between two conditions were identified through fold change filtering. Heatmaps and

Table 2 Pregnancy complications

\begin{tabular}{llll}
\hline Characteristics & Oligohydramnios $(\mathbf{n}=\mathbf{2 0 )}$ & $\begin{array}{l}\text { Normal control } \\
(\mathbf{n = 1 9 )}\end{array}$ & P-value \\
\hline FGR (\%) & $7(35)$ & 0 & $0.005^{\#}$ \\
RSA (\%) & $5(25)$ & 0 & $0.027^{\#}$ \\
Premature birth (\%) & $6(30)$ & 0 & $0.012^{\#}$ \\
GDM (\%) & $1(5)$ & $2(10.5)$ & 0.48 \\
UCTD (\%) & $1(5)$ & 0 & 0.513 \\
Mild anemia (\%) & $2(10)$ & 0 & 0.256 \\
Hypothyroidism (\%) & $2(10)$ & $4(21.1)$ & 0.305 \\
Thalassemia (\%) & $2(10)$ & 0 & 0.256 \\
\hline
\end{tabular}

FGR Fetal growth restriction, RSA Recurrent abortion, GDM Gestational diabetes mellitus, UCTD Undifferentiated connective tissue disease. * $P<0.05$ (Student's ttest). Classification variable is calculated by ratio (\%). "Fisher was used to accurately test $P$-value $<0.05$ 
scatter plots were generated for differentially expressed genes using the $\mathrm{R}$ package (version 3.1.0) [19]. Kyoto Encyclopedia of Genes and Genomes (KEGG) pathway analysis was performed using an online tool (http:// www.genome.jp/kegg/). KEGG pathways that met the requirement of False Positive Discovery (FDR) $\leq 0.001$ were considered significantly enriched.

To explore the potential role of lncRNA, a lncRNAmiRNA-mRNA interaction network was constructed. We used miRNA target gene prediction software (miRanda) to predict miRNA targets on IncRNA. The overlap miRNAs that harbored both lncRNA and mRNA binding targets were used to construct the IncRNAmiRNA-mRNA interaction network. The sub-network that contained predicted targets of IncRNA and was differentially expressed in OP was included. The network was visualized using Cytoscape_V2_8_3 (https://www. innatedb.ca/cytoscape-v2.8.3/plugins/) software.

\section{qPCR}

The relative expression of IncRNA between 20 OP and 19 Normal was measured by qPCR. Total RNA was reverse transcribed to cDNA using PrimeScript RT Master Mix (Takara, Dalian, China). cDNAs were then amplified and quantified on an ABI 7500 real-time PCR system (Applied Biosystems, CA, USA) with a SYBR Real time PCR Master Mix Kit (TOYOBO, Osaka, Japan). The program for cDNA amplification was as follows: the first step, $95^{\circ} \mathrm{C}$ for $120 \mathrm{~s}$; the second step, $95^{\circ} \mathrm{C}$ for $15 \mathrm{~s}$ and $60^{\circ} \mathrm{C}$ for $30 \mathrm{~s}$, for 40 cycles; the third step, for melting curve generation, $60^{\circ} \mathrm{C}$ to $95^{\circ} \mathrm{C}$. The relative expression of IncRNA was analyzed using the $2^{-\Delta \Delta \mathrm{Ct}}$ method. GAPDH was used as an internal control. The primers were shown in Table 3.

\section{Statistical analysis}

Student's t-test was used to analyze the significant differences by the SPSS 18.0 software package in the study. Three biological replicates were performed in the study. A $P$-value of $<0.05$ defined the significant differences between the two groups.

Table 3 Primers used in this study

\begin{tabular}{llc}
\hline Primer name & Sequence (5' to $\mathbf{3}^{\prime}$ ) & Production size \\
\hline LINC00515F & TCAAGGCAGCAGTGGCAGAG & 142 \\
LINC00515R & AGTCACAGGCGTGGAGGTCA & \\
RP11-388P92F & ATTTGCCAGCTTCTCCTTTGA & 145 \\
RP11-388P92R & TTGGCAGAATGAGACATCAAG & \\
GAPDHF & GAGTCAACGGATTTGGTCGT & 185 \\
GAPDHR & GAGTCAACGGATTTGTCGT & \\
\hline
\end{tabular}

\section{Results}

\section{Overview of IncRNA and mRNA profiles in OP}

There was no significant difference in the age, number of pregnancy, and number of productions between the two groups of pregnant women (Table 1). The weeks of delivery and number of spontaneous abortion in the OP were $37.34 \pm 2.01$ and $0.7 \pm 0.92$, respectively, which were significantly lower than those of Normal group (Table 1). The main delivery methods in OP and Normal groups were cesarean section (60\%) and normal delivery (68.4\%), respectively (Table 1 ). The birth weight and placental weight in OP group were $2.67 \pm 0.57$ and $0.47 \pm 0.04 \mathrm{~kg}$, respectively, which were significantly lower than those of the Normal group (Table 1). Some pregnant women with oligohydramnios had symptoms of gestational diabetes mellitus (GDM), undifferentiated connective tissue disease (UCTD), mild anemia, hypothyroidism, and thalassemia (Table 2). However, there were only 2 cases of gestational diabetes mellitus and 4 cases of hypothyroidism in the Normal group (Table 2).

To explore the profile of lncRNA and mRNA in the fetal membranes of five OP and five Normal were obtained and subjected to microarray analysis. As shown in Fig. 1, the profiles of lncRNA and mRNA in OP were different from those in Normal. A sequence of lncRNAs and mRNAs were alert in OP, compared with Normal (Fig. 1a\&b). Among the differentially expressed lncRNAs, 638 were upregulated and 163 were downregulated (Fig. 1c and Supplemental Table 1). Of the differentially expressed mRNAs, 189 were upregulated and 178 were downregulated, as shown in the volcano plot (Fig. 1d and Supplemental Table 2).

\section{Characteristics of the differentially expressed IncRNAs and mRNAs in OP}

To further observe the expression characteristics of these differentially expressed lncRNAs and mRNAs, there was an analysis of the genomic location distribution as well as the length and type distribution. The statistical results of the differentially expressed lncRNA showed that the differentially expressed lncRNA was mainly distributed on chromosomes 2, 1, and 11, with the least distribution on the Y chromosome; this indicated that the IncRNAs that played a role in oligohydramnios were mainly located on chromosomes 2, 1 and 11 (Fig. 2a). The length distribution showed that most lncRNAs were distributed within $1 \mathrm{~kb}$, whereas the mRNAs were mainly distributed at $2-3 \mathrm{~kb}$ in length (Supplemental Figure 1). Analysis for differential lncRNA type revealed the largest number of intergenic lncRNAs, followed by intronic antisense and natural antisense, indicating that the lncRNAs that played a role in oligohydramnios are mainly intergenic lncRNAs (Fig. 2b). KEGG results revealed that differential mRNAs 

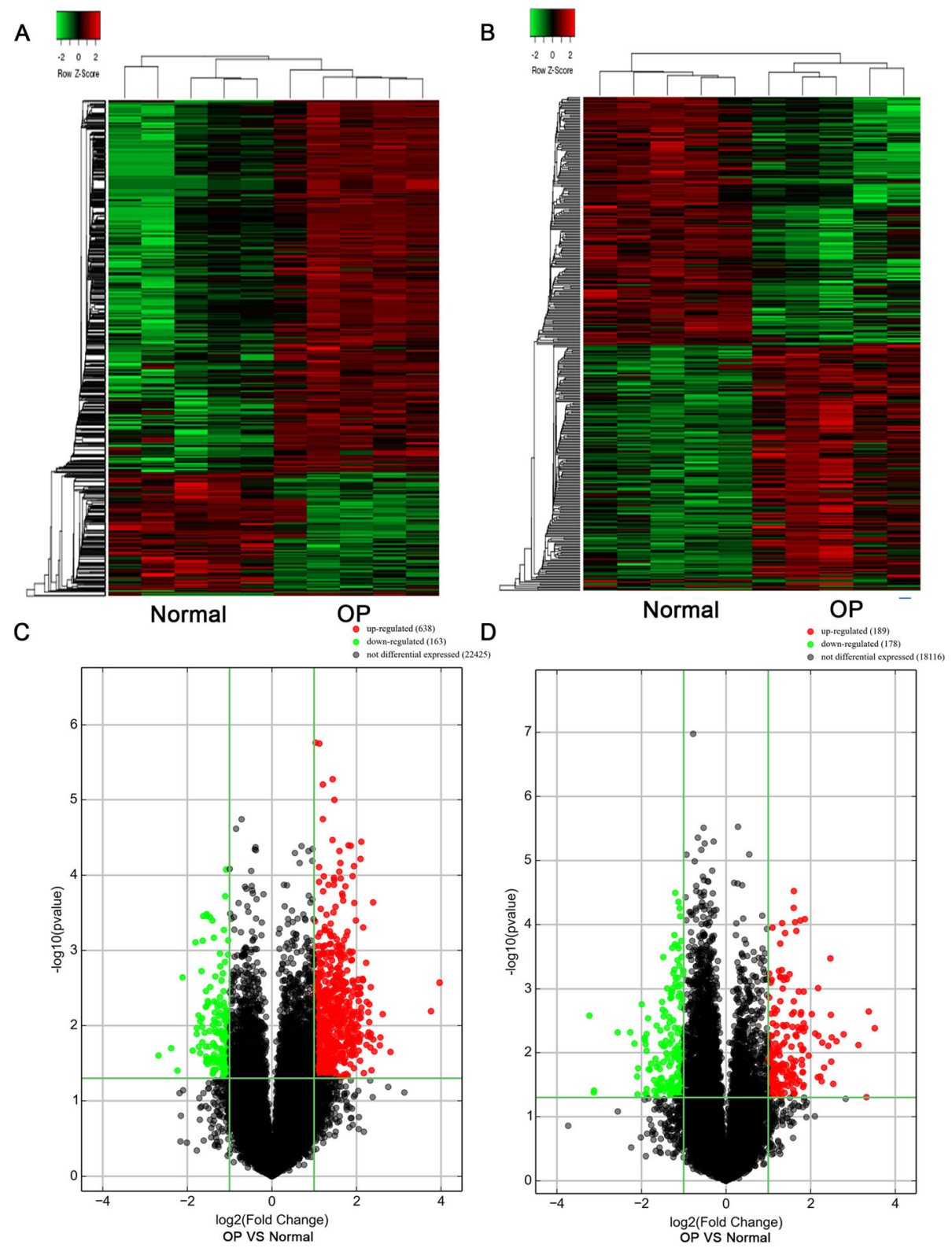

Fig. 1 Microarray analysis revealed differential IncRNA profiles in fetal membranes resected from five oligohydramnios pregnant women (OP) and five normal amount of amniotic fluid pregnant women (Normal). The heatmap showed the profile of IncRNA (a) and mRNAs (b) in OP and Normal. The volcano plot showed the overall change in expression of IncRNAs (c) and mRNAs (d). The upregulated RNA is labeled in red, whereas the downregulated RNA is labeled in green

mainly enrich in focal adhesion as well as in the signaling pathways of Ras, tumor necrosis factor (TNF), estrogen, and chemokine (Fig. 3). Other top pathways were shown in Fig. 3.

The expression of IncRNAs and the potential regulatory network

In order to explore the function of differentially expressed lncRNAs in OP, specific analysis was conducted for the differentially expressed lncRNAs and their regulatory network. Table 4 listed the lncRNAs in the top 10 of upregulated and downregulated expression in OP women. As shown in Table 4, the highest differential expression was G017197, and the upregulation fold change was 6.99 times; the highest downregulation was G083088, and the expression was downregulated to 0.15 -fold. We verified the differential expression of two specific lncRNAs, LINC00515 and RP11-388P9.2, by using qPCR (Fig. 4). As shown in Fig. 4, both LINC00515 and RP11-388P9.2 showed increased 

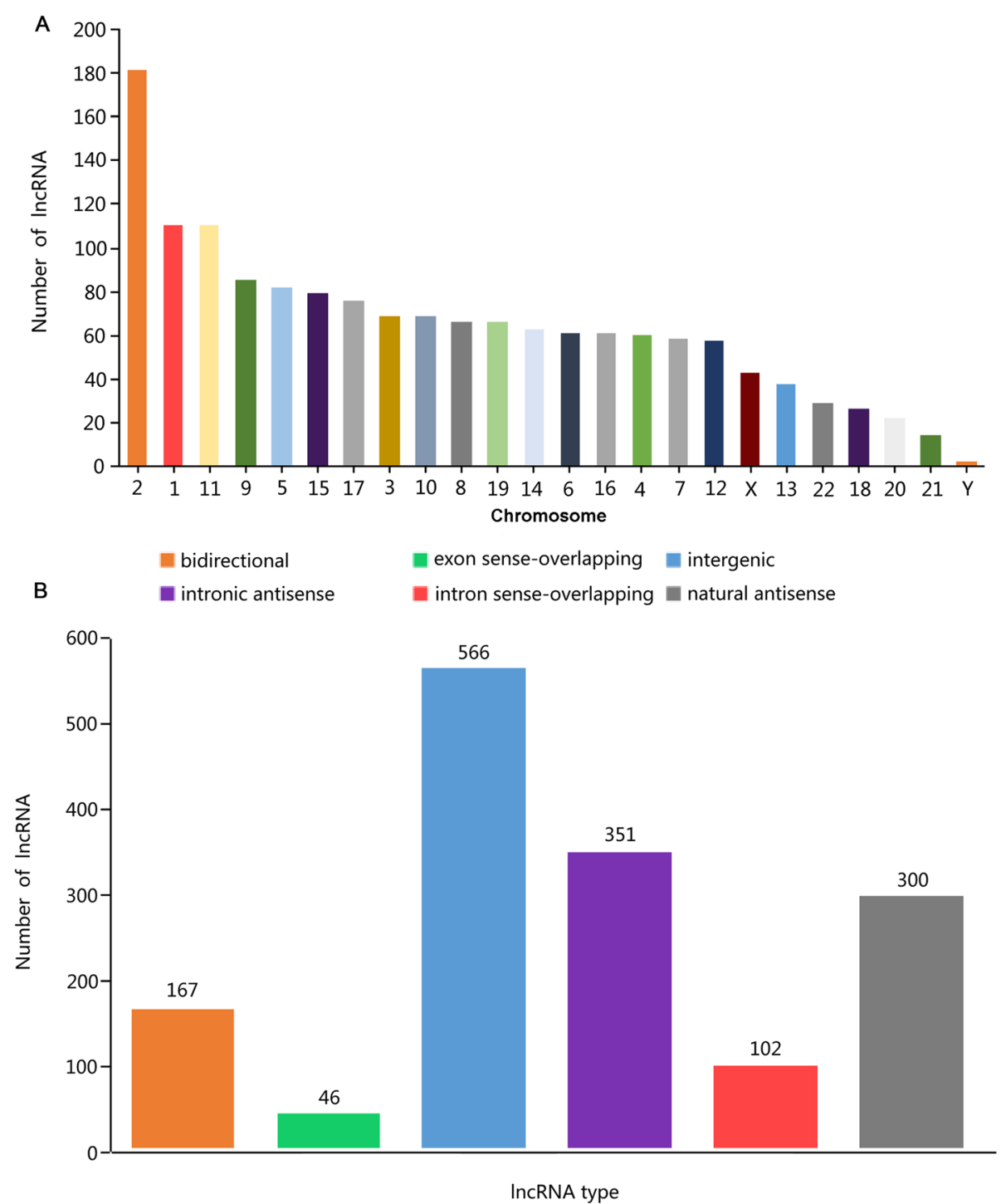

Fig. 2 Distribution of IncRNA genomic location (a) and type of the differentially expressed IncRNA (b) in oligohydramnios pregnant women (OP)

expression in OP. Furthermore, a lncRNA-miRNAmRNA interaction network based on LINC00515 and RP11-388P9.2 was generated. Potential miRNA targets of LINC00515 and RP11-388P9.2 were predicted and then screened for consistency with mRNAs that were expressed as upregulated in OP. As revealed in Fig. 5, a regulatory network of LINC00515 and RP11-388P9.2 was obtained. The network included 27 miRNAs and 5 mRNAs (Fig. 5). The mRNAs that were finally captured were tenascin $R$ (TNR), cystic fibrosis transmembrane conductance regulator (CFTR), ATP-binding cassette sub-family A member 12 (ABCA12), and collagen 9A2 (COL9A2).

\section{Discussion}

Oligohydramnios is one of the common obstetric complications. The etiology of oligohydramnios mainly includes the fetal factor, placental membrane factor, maternal factor, and drug factor. For example, in the prolonged pregnant women with oligohydramnios, resistance index in the fetal renal artery is higher than it is in the controls, which are without oligohydramnios [20]; additionally, cyclooxygenase- 2 inhibitor nimesulide and long-term diclofenac exposure are associated with oligohydramnios $[21,22]$. In our study, the possible causes of oligohydramnios in pregnant women may be due to an increased number of abortions and pregnancy complications; still, the underlying molecular mechanism for oligohydramnios remains unknown. Therefore, we conducted microarray analysis and unveiled the expression profile of lncRNA and mRNA in OP. The qPCR results confirmed that LINC00515 and RP11-388P9.2 were upregulated in OP. IncRNA-miRNA-mRNA regulatory 


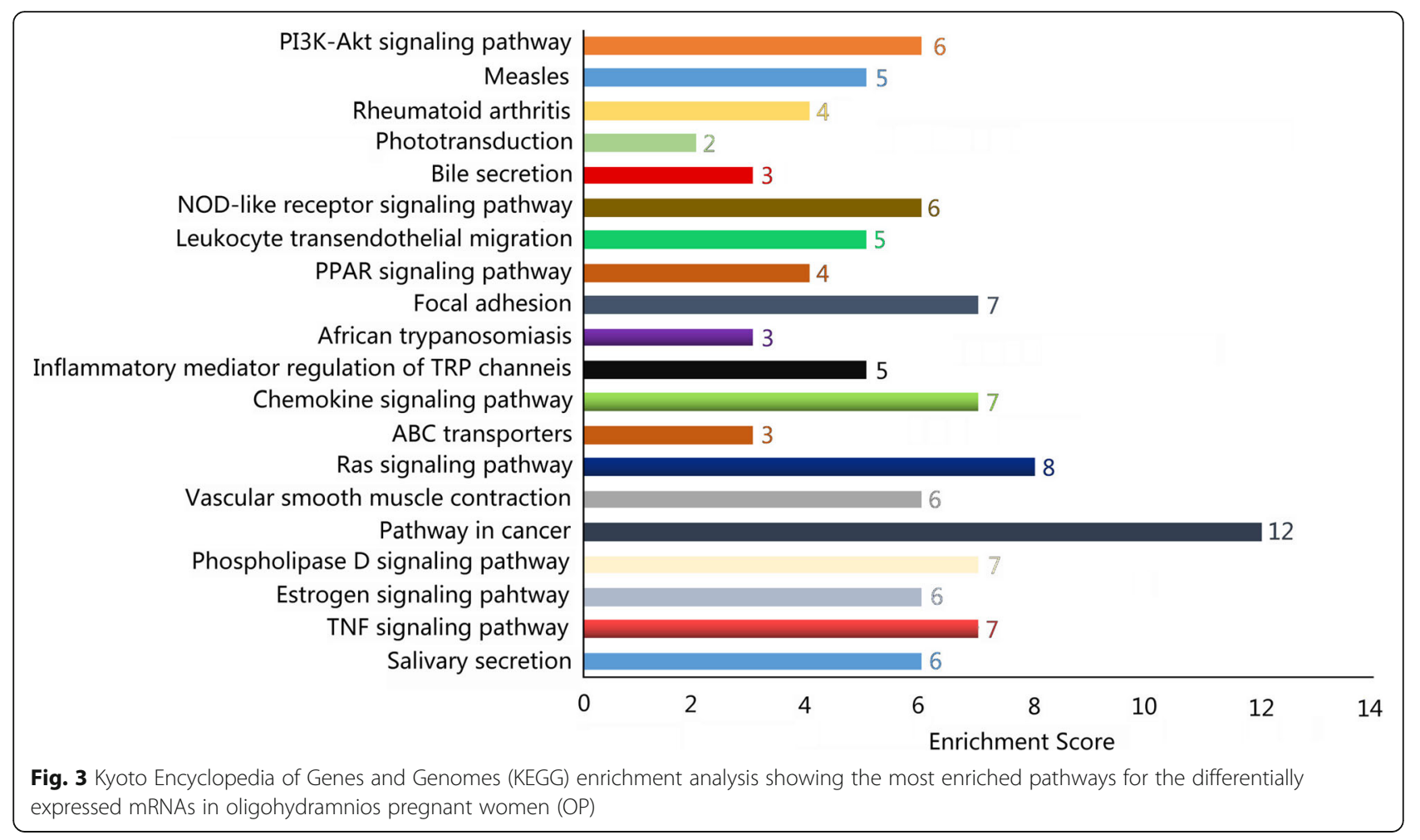

Table 4 Information of the top-10 most upregulated and downregulated IncRNAs in oligohydramnios pregnant women (OP)

\begin{tabular}{|c|c|c|c|c|}
\hline IncRNA_ID & Fold Change & Regulation & P-value & FDR \\
\hline G017197 & 6.992997551 & up & 0.022330367 & 0.323992752 \\
\hline G028960 & 6.156767122 & up & 0.007011989 & 0.231007727 \\
\hline G056426 & 5.925028116 & up & 0.014297859 & 0.282223415 \\
\hline GSE61474_XLOC_033346 & 5.875790672 & up & 0.018248847 & 0.30550168 \\
\hline G059353 & 5.369031987 & up & 0.022024259 & 0.322125587 \\
\hline AC091729.7 & 5.280611483 & up & 0.015183552 & 0.286476988 \\
\hline LINC00515 & 5.265016308 & up & 0.000229591 & 0.092301652 \\
\hline G024752 & 5.123561472 & up & 0.039183093 & 0.394165858 \\
\hline RP11-388P9.2 & 5.0709114 & up & 0.01273173 & 0.271540086 \\
\hline DPP10-AS1 & 5.0609152 & up & 0.003061877 & 0.185019347 \\
\hline G083088 & 0.156292992 & down & 0.024924406 & 0.335128165 \\
\hline LINC00501 & 0.192207162 & down & 0.019901778 & 0.31149042 \\
\hline G045291 & 0.213060492 & down & 0.039570275 & 0.395804997 \\
\hline LINC01510 & 0.231630157 & down & 0.002294572 & 0.176676707 \\
\hline RP11-1399P15.1 & 0.273437307 & down & 0.021416751 & 0.321541985 \\
\hline RP11-150012.1 & 0.286446378 & down & 0.000781865 & 0.143720717 \\
\hline LRRC74B & 0.292328015 & down & 0.010723383 & 0.257909493 \\
\hline RP11-567G11.1 & 0.292488539 & down & 0.012877268 & 0.272737873 \\
\hline G016402 & 0.301039562 & down & 0.007261346 & 0.232753219 \\
\hline G043293 & 0.302661694 & down & 0.019071938 & 0.309549149 \\
\hline
\end{tabular}

FDR False positive discovery 


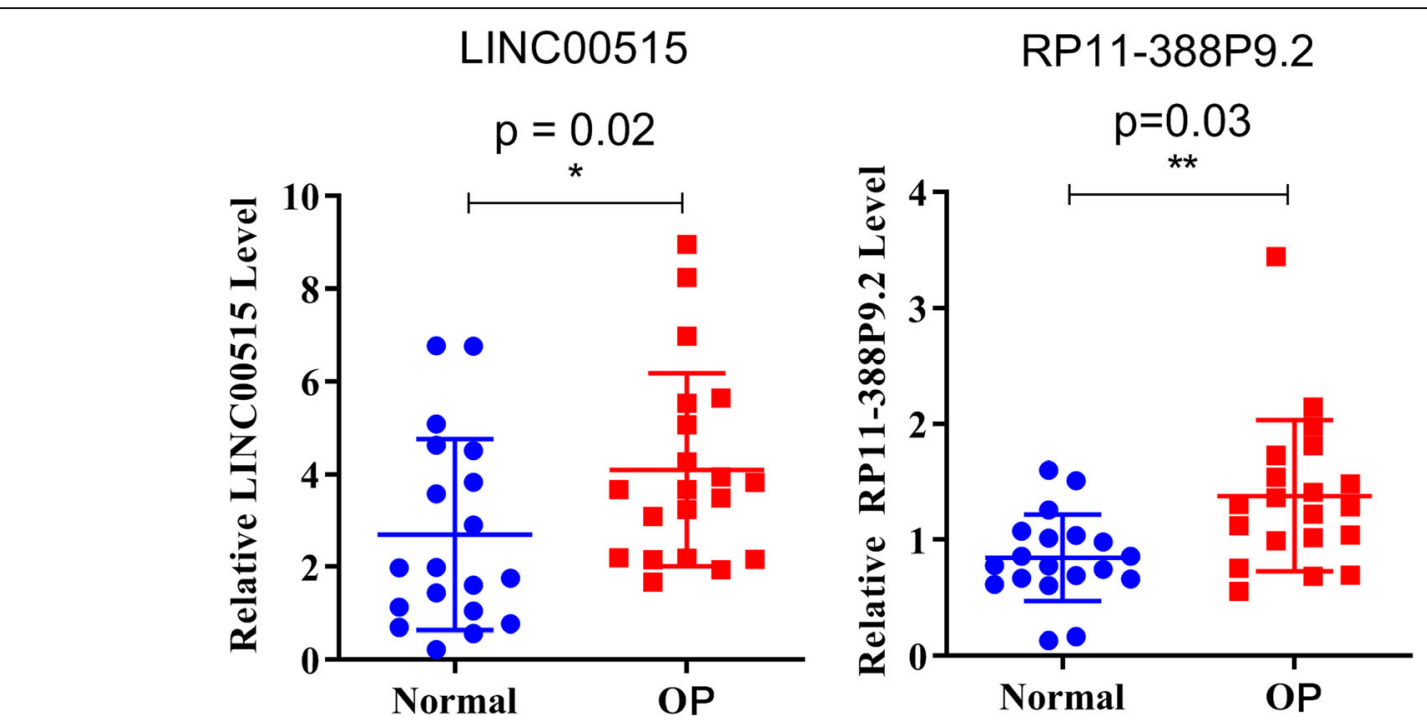

Fig. 4 The expression of LINC00515 and RP11-388P9.2 in oligohydramnios pregnant women (OP) and normal amount of amniotic fluid pregnant women (Normal). ${ }^{*} P<0.05$ represents the significant difference

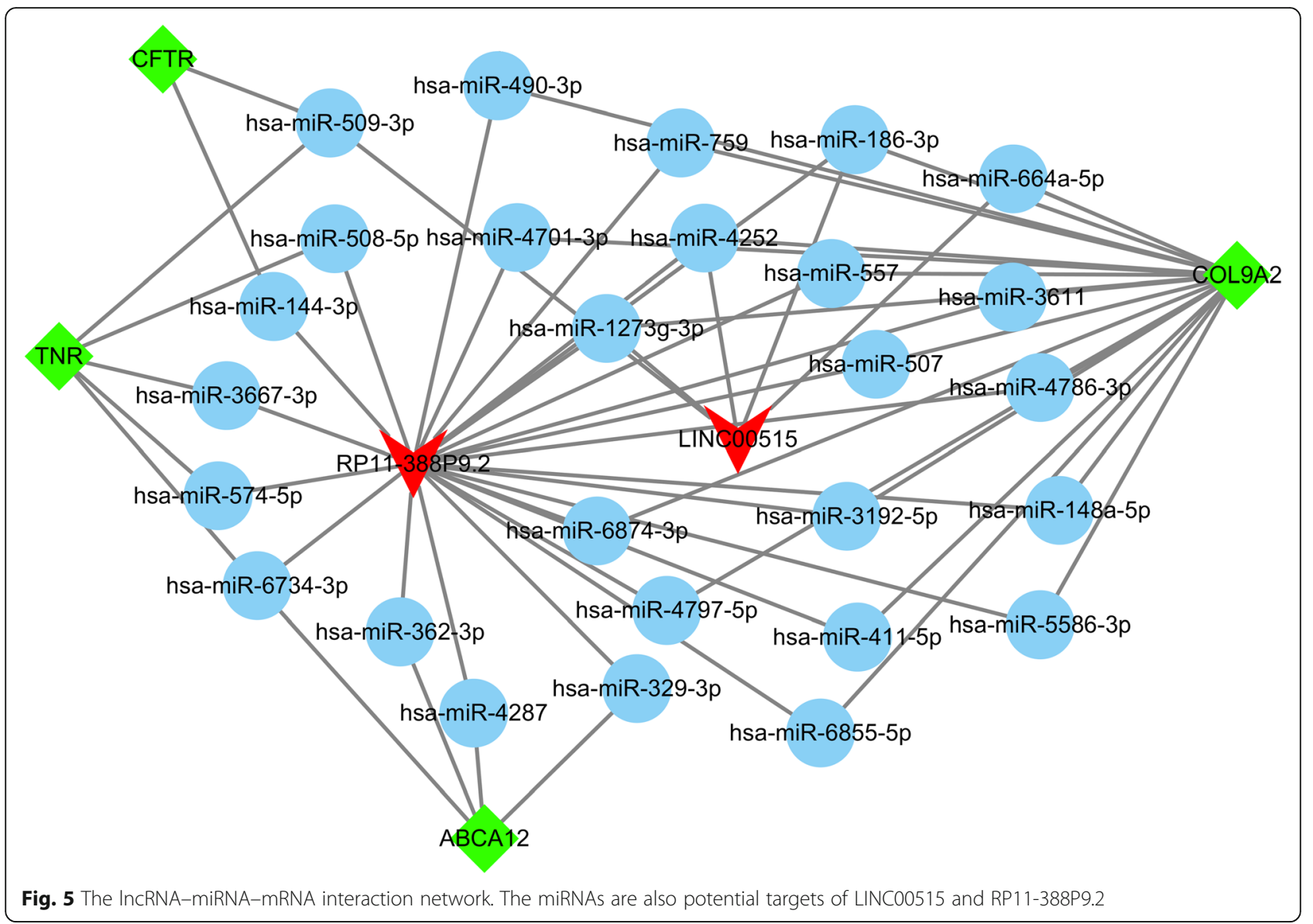


network revealed TNR, CFTR, ABCA12, and COL9A2 as the candidate targets of LINC00515 and RP11388P9.2. These results showed that the expression of lncRNAs in fetal membrane tissues might plays an important role in pregnant women with oligohydramnios.

The fetal membrane is a vital tissue for communication between mother and fetus. There is a hypothesis that the resorption pathway that crosses the amnion to the fetal circulation may keep the balance of normal amniotic fluid volume [20]. Therefore, it is reasonable to speculate that changes in the expression of molecules in the membrane tissue are response to changes in the microenvironment. Many studies have previously provided the molecular information [23-26] of the fetal membrane tissue. The expression of aquaporin 8 and aquaporin 9 in amniotic membrane of oligohydramnios group is significantly lower than that of normal amniotic fluid group, however, it has not been shown which lncRNAs regulate the expression of aquaporin 8 and aquaporin 9 in oligohydramnios group [27]. Simultaneously, the expression analysis of lncRNA and mRNA in the fetal membrane tissue of pregnant women with oligohydramnios is still limited. Here we showed that 638 lncRNAs and 189 mRNAs were upregulated and that 163 lncRNAs and 178 mRNAs were downregulated. Moreover, we found that the differentially expressed mRNAs were mainly enriched in focal adhesion as well as in the signaling pathways of Ras, TNF, estrogen, and chemokine. Research shows that TNF- $\alpha$ and interleukin 6 (IL-6) play an essential role in the inflammatory process in pregnant women with spontaneous preterm births [28]. Under the induction of ferutinin, human amniotic fluid stem cells are differentiated into osteoblasts through estrogen receptor $\alpha$ and the phosphatidylinositol 3-kinase (PI3K)/protein kinase B (Akt) signal pathway [29]. The TNF signaling pathway and the estrogen signaling pathway may be involved in the regulation of OP.

We also confirmed the upregulation of two lncRNAs, LINC00515 and RP11-388P9.2, in OP. Also, a lncRNAmiRNA-mRNA interaction network was constructed to illustrate the possible function of LINC00515 and RP11388P9.2 involved in oligohydramnios. In the network, the role of almost all miRNAs in oligohydramnios remains unknown. In addition, four mRNAs, CFTR, TNR, ABCA12, and COL9A2 were predicted to be included in the regulatory network of LINC00515 and RP11388P9.2. Of these mRNAs, CFTR, a small conductance $\mathrm{Cl}^{-}$channel, is regulated by intracellular ATP and cAMP-dependent phosphorylation, predominantly located in the apical membrane of organ epithelial cells. The study of endometrial epithelia shows that CFTR is involved in the secretory effects of ovarian hormone regulation [30], showing that CFTR plays a vital role in female reproduction. It can be inferred that RP11-
388P9.2 might affect the CFTR expression by regulating has-miR-114-3p, further affecting the amniotic fluid content of pregnant women. Moreover, TNR can interact with fibronectin 1, which can be involved in cell migration and adhesion biological processes including embryogenesis [31]. Tenascin is also produced in the extracellular matrix of cultured amnion epithelial cells [32]. It can be inferred that RP11-388P9.2 might affect the TNR expression by regulating the has-miR-508-5p, has-miR-3667-3p, has-miR-574-5p, and has-miR-6734$3 p$, further affecting the amniotic fluid content of pregnant women. Compared with the amniotic fluid cells of a healthy human, the cells of fetuses with neural tube defects do not deposit type I collagen [33]. It can be inferred that LINC00515 might affect the COL9A2 expression by regulating the has-miR-186-3p and has-miR664a-5p, further affecting the amniotic fluid content of pregnant women.

Based on the above deduction, we could conclude that LINC00515 and RP11-388P9.2 regulated the expression of different miRNAs to control the expression levels of TNR, CFTR, ABCA12, and COL9A2, which affected the decrease of amniotic fluid content in pregnant women. However, their mechanism is still unclear and needs further study.

\section{Conclusions}

In summary, we revealed the profiles of lncRNA and mRNA in OP, validated the upregulation of LINC00515 and RP11-388P9.2, and suggested a IncRNA-miRNAmRNA network that might be involved in the pathogenesis of oligohydramnios. These results might offer potential targets for biological prevention for pregnant women with oligohydramnios detected before delivery.

\section{Supplementary information}

Supplementary information accompanies this paper at https://doi.org/10. 1186/s12920-020-00792-z.

Additional file 1: Figure S1. Distribution of IncRNA length in oligohydramnios pregnant women (OP).

Additional file 2: Table S1. Differentially expressed IncRNAs between group-normal amount of amniotic fluid pregnant women (Normal) and the group-oligohydramnios pregnant women (OP).

Additional file 3: Table S2. Differentially expressed mRNAs between group-normal amount of amniotic fluid pregnant women (Normal) and the group-oligohydramnios pregnant women (OP).

\section{Abbreviations}

LncRNAs: Long noncoding RNAs; OP: Oligohydramnios pregnant women; Normal: Normal amount of amniotic fluid pregnant women; KEGG: Kyoto Encyclopedia of Genes and Genomes; qPCR: Real-time quantitative PCR; TNF: Tumor necrosis factor; TNR: Tenascin R; CFTR: Cystic fibrosis

transmembrane conductance regulator; $A B C A 12$ : ATP-binding cassette subfamily A member 12; COL9A2: Collagen 9A2; AFI: Amniotic fluid index; SDP: Single deepest pocket; qPCR: Real-time quantitative PCR; PBS: Phosphate buffered saline; RPKM: Reads per kilobase of transcript per million reads mapped; FDR: False Positive Discovery; GDM: Gestational 
diabetes mellitus; UCTD: Undifferentiated connective tissue disease; IL6: Interleukin 6; PI3K: Phosphatidylinositol 3-kinase; Akt: Protein kinase B

\section{Acknowledgments}

Not applicable.

\section{Authors' contributions}

$\mathrm{HC}$ and JPZ were responsible for the study design. YHO and YKL wrote the manuscript. JPZ edited and corrected the manuscript. YHO, YKL and LQZ were responsible for the experimental studies. $\mathrm{YHO}, \mathrm{HC}, \mathrm{MQC}$, and $\mathrm{XCY}$ were responsible for the integrity of the data and the accuracy of the data analysis. MQC and XCY were responsible for participant recruitment, tissue sample collection, and clinical data collation. All authors have read and approved the manuscript.

\section{Funding}

This study was supported by the National Nature Science Foundation of China (81771660) given to Jian-ping Zhang for microarray analysis, Guangdong Natural Science Foundation (2018A030313023) given to Hui Chen for obtaining qPCR detection reagents and RNA extraction reagents, Guangdong Natural Science Foundation (2018A030310162) given to Li-giong Zhu for obtaining qPCR detection reagents and RNA extraction reagents, and Major Program of the National Natural Science Foundation of China (2019YFA0801403) given to Jian-ping Zhang for obtaining liquid nitrogen tank

\section{Availability of data and materials}

The datasets used and analyzed during the current study are available from the corresponding author on reasonable request. The datasets generated during the current study are available in the GEO Database Series GSE142701 repository, [https://www.ncbi.nlm.nih.gov/geo/query/acc.cgi?acc=GSE142701].

\section{Ethics approval and consent to participate}

Written informed consent was obtained from all pregnant women. This study was approved by the Ethics Committee of Sun Yat-sen Memorial Hospital, Sun Yat-sen University.

\section{Consent for publication}

Written informed consent was obtained from all pregnant women.

\section{Competing interests}

The authors declare no conflict of interest

\section{Author details}

'Department of Obstetrics and Gynecology, Guangdong Women and Children Hospital, Guangzhou 511400, Guangdong, China. ${ }^{2}$ Department of Obstetrics and Gynecology, Sun Yat-sen Memorial Hospital, Sun Yat-sen University, No.107, Yanjiangxi Road, Guangzhou 510120, Guangdong, China.

Received: 9 August 2019 Accepted: 3 September 2020

\section{Published online: 18 September 2020}

\section{References}

1. Beall MH, et al. Regulation of amniotic fluid volume. Placenta. 2007;28(8): 824-32.

2. Ounpraseuth ST, et al. Normal amniotic fluid volume across gestation: comparison of statistical approaches in 1190 normal amniotic fluid volumes J Obstet Gynaecol Res. 2017:56(4 Pt 1):11.

3. Lei H, Wen SW. Normal amniotic fluid index by gestational week in a Chinese population. Central-South China fetal growth study group. Obstet Gynecol. 1998;92(2):237-40.

4. Gadd RL. The volume of the liquor amnii in normal and abnormal pregnancies. J Obstet Gynaecol British Commonwealth. 2010;73(1):11-22.

5. Morris JM, et al. The usefulness of ultrasound assessment of amniotic fluid in predicting adverse outcome in prolonged pregnancy: a prospective blinded observational study. Bjog. 2003;110(11):989-94.

6. Magann EF, et al. The amniotic fluid index, single deepest pocket, and twodiameter pocket in normal human pregnancy. Am J Obstet Gynecol. 2000; 182(6):1581-8
7. Shrem $\mathrm{G}$, et al. Isolated Oligohydramnios at term as an indication for labor induction: a systematic review and meta-analysis. Fetal Diagn Ther. 2016: 40(3):161-73.

8. Rabie $\mathrm{N}$, et al. Oligohydramnios in complicated and uncomplicated pregnancy: a systematic review and meta-analysis. Ultrasound Obstet Gynecol. 2017:49(4):442-9.

9. Casey BM, et al. Pregnancy outcomes after antepartum diagnosis of oligohydramnios at or beyond 34 weeks' gestation 2 ? Gynecol. 2000;182(4):909-12.

10. Asnafi N, Bouzari Z, Mohammadnetadj M. Oligohydramnios and pregnancy outcome: ten-years review. Int Biol Biomed J. 2015:1(1):23-8.

11. Tarannum K, et al. Oligohydramnios and fetal outcome: a review. Med Phoenix. 2016;1(1):23-30.

12. Huarte M. The emerging role of IncRNAs in cancer. Nat Med. 2015;21(11):1253.

13. Li N, et al. The role of MicroRNA and LnCRNA-MicroRNA interactions in regulating ischemic heart disease. J Cardiovasc Pharmacol Therapeutics. 2016;22(2):105-11.

14. El AH, Doevendans PA, Sluijter JP. Long non-coding RNAs in heart failure: an obvious Inc. Ann Transl Med. 2016;4(9):182.

15. Wang J, Wei F, Zhou H. Advances of IncRNA in autoimmune diseases. Front Laboratory Med. 2018;2(2):79-82.

16. Machtinger $\mathrm{R}$, et al. Placental IncRNA expression is associated with prenatal phthalate exposure. Toxicol Sci Official J Soc Toxicology. 2018;163(1):116.

17. Zhang $P$, et al. LncRNA uc003fir promotes CCL5 expression and negatively affects proliferation and migration of trophoblast cells in preeclampsia. Pregnancy Hypertension ER. 2018;14:90-6.

18. Zhu XQ et al. Expression of aquaporin 1 and aquaporin 3 in fetal membranes and placenta in human term pregnancies with oligohydramnios. Placenta. 2009;30(8):670-6.

19. Wang $X$, et al. Whole-genome sequencing of eight goat populations for the detection of selection signatures underlying production and adaptive traits. Sci Rep. 2016:6:38932.

20. Akram $\mathbf{W}$. The role of resistance index in fetal renal artery in causing oligohydramnios among overdue pregnant women. Mustansiriya Med J. 2014;13(1):2018

21. Holmes RP, Stone PR. Severe oligohydramnios induced by cyclooxygenase-2 inhibitor nimesulide. Obstet Gynecol. 2000;96(5):810-1.

22. Scherneck $\mathrm{S}$, et al. Reversible oligohydramnios in the second trimester of pregnancy in two patients with long-term diclofenac exposure. Reprod Toxicol. 2015;58:61-4.

23. Enquobahrie DA, et al. Candidate gene and MicroRNA expression in fetal membranes and preterm delivery risk. Reprod Sci. 2016;23(6):731.

24. Ramkumar $\mathrm{M}$, et al. Expression of 8-oxoguanine glycosylase in human fetal membranes. Am J Reprod Immunol. 2014;72(1):75-84.

25. Michelle B-F, et al. Differential expression of the enzymatic system controlling synthesis, metabolism, and transport of PGF2 alpha in human fetal membranes. Biol Reprod. 2001;83(1):155-62.

26. Pereyra $\mathrm{S}$, et al. Transcriptomic analysis of fetal membranes reveals pathways involved in preterm birth. BMC Med Genomics. 2019;12(1):53.

27. Jiang SS, et al. Expression and localization of aquaporins 8 and 9 in term placenta with oligohydramnios. Reprod Sci. 2012;19(12):1276-84.

28. Melekoglu R, et al. Associations between second-trimester amniotic fluid levels of ADAMTS4, ADAMTS5, IL-6, and TNF-alpha and spontaneous preterm delivery in singleton pregnancies. J Perinat Med. 2019;47(3):304-10.

29. Zavatti $M$, et al. Estrogen receptor signaling in the ferutinin-induced osteoblastic differentiation of human amniotic fluid stem cells. Life Sci. 2016;164:15-22

30. Jin $\mathrm{L}$, Tang R. Expression of cystic fibrosis transmembrane conductance regulator in rat ovary. J Huazhong Univ Sci Technol Med Sci. 2008;28(5):584-7.

31. Hawi $Z$, et al. A case-control genome-wide association study of ADHD discovers a novel association with the tenascin R (TNR) gene. Trans Psychiatry. 2018:8(1):284

32. Linnala A, von Koskull H, Virtanen I. Isoforms of cellular fibronectin and tenascin in amniotic fluid. FEBS Lett. 1994;337(2):167-70.

33. Hosper NA, R.A. Bank, van den Berg PP. Human amniotic fluid-derived mesenchymal cells from fetuses with a neural tube defect do not deposit collagen type i protein after TGF-beta1 stimulation in vitro. Stem Cells Dev. 2014;23(5):555-62.

\section{Publisher's Note}

Springer Nature remains neutral with regard to jurisdictional claims in published maps and institutional affiliations. 